\title{
Embracing digital: Key considerations for publishers, marketers and customers
}

\author{
Rosalind Hill \\ Digital Publishing Director, Future Science Group, Unitec House, 2 Albert Place, N3 1QB, UK \\ Tel.: 0044208371 6090; E-mail: r.hill@futuremedicine.com
}

\begin{abstract}
As publishers and our customers embrace the opportunities that digital can offer, there are key considerations for product and content development that should be taken into account to enrich user journeys and provide customers with relevant content at the right time. In this article, the author discusses these key considerations in light of shifting user demographics, technology developments, and digital trends. For time-pressed individuals, the ability to quickly and easily access information is paramount and publishers have a variety of tools at their fingertips through which to provide relevant content, but the fight for attention is growing and navigating the landscape can be confusing.
\end{abstract}

Keywords: Digital, mobile technology, millennials, publishing, search engine optimization, video, social media

\section{Introduction}

The publishing landscape has changed significantly over the last decade alone, with a huge shift towards digital tools and technologies. Publishers have to adapt the ways in which they reach their audience with the content they need, when they need it. This means embracing digital technologies beyond traditional print journals and online journal platforms.

For many, however, digital has become a minefield, and simply creating a website or launching a Twitter account won't get the engagement required from the end user to succeed. We're in an age in which we're not just jostling with direct competitors for space, but with international news outlets, bloggers, and more. The noise can be deafening, but there are ways to overcome it, and the key to that is understanding the shifting demographics of our end users and the trends that are driving digital forward.

\section{Shifting demographics of the end user}

Until relatively recently, the workforce was dominated by Baby Boomers (born 1946-1964) and Generation X (1965-1980). The Baby Boom generation can be characterized by the Swinging 60s and the Cold War; they're in favour of equal opportunity and job security. When it comes to technology they're early adopters and increasingly stay in touch through social media platforms such as Facebook. However, generally their preferred methods of communication remain via face-to-face meetings or telephone. Generation X aspired for the ideal in work-life balance and technology was easily assimilated into their daily life; they grew up as the World Wide Web and internet developed, were using PCs at school and generally prefer email and text over telephone or face-to-face communications. 
As the year 2000 approached, Millennials (1981-1995) began to enter the workforce and today make up $27 \%$ of the global population. A digitally-native group, they are tech-forward and characterized by the tablet and smart phone. They like freedom and flexibility when it comes to work and generally communicate via social media channels such as Facebook, Instagram or Snapchat. Connectivity is everything and they earn to spend. Millennials have been the driving force of much digital advancement; Mark Zuckerberg, for example, was born in 1984 and is a founder of Facebook. Even the creators of Google are only in their early 40s. Both Facebook and Google have driven the consumption of information past the subscription to newspapers, magazines, and journals to touch-of-a-button access and sharing.

In 2013, Generation Z (born 1995-2010) began to enter the workforce, and this will be a key gamechanger for the publishing industry of the future. This group is characterized by the economic downturn, mobile technology, global warming, the Arab (and indeed, Academic) Spring, Cloud computing and WikiLeaks; a generation for which instant gratification is the norm. Their signature products are nanocomputing, 3D printing, and the Google Glass. They prefer to talk online rather than in person and are technoholics - they have a limited grasp of the alternatives. Publishers and the way in which research and the written word is shared will need to adapt to meet this generation's needs. They will need to be tech-savvy, digitally-native, and social networkers.

All these different generations combine to make an interesting landscape through which publishers have to navigate, and it is now more important than ever that we take the user on a journey that works for each generation.

\section{What's important to end users?}

My discussions with, and feedback from, various members of the scientific and medical communities, identified the pain points with which we are all too familiar:

- Open access and the legal challenges of accessing research

- The integration of big, diverse datasets

- Solo and silo working

- Data protection concerns

- Poor communication

- Time

When asked what is important to these communities, unsurprisingly, access to information is paramount. This includes the curation of relevant information and content, as well as information on unpublished data. Networking with peers and other specialties are also important - as are customized solutions.

These attitudes correlate with the various research into the online behaviours of users across a variety of specialties, professional and consumer. It has never been more apparent that the customer is king; a king who wants personalization and targeted content - when he/she wants it. In the consumer industry, one might visit an online store searching for a particular item, or search the web for information on a particular product - the user expects this information to be provided at the touch of a button. If it is too difficult to find what they're looking for, then the search is often abandoned, or a brand is left with a black mark against its name. Likewise, in the professional publishing industry, our users do not want to search through huge amounts of data and content to find what they're looking for - they will often have a very clear intention for their online journey. 
Ninety-three percent of online journeys start with a search engine and it is predicted that by 2020 , $50 \%$ of all search will be voice or image driven [5]. Even now, it's estimated that $70 \%$ of Millennials use voice search $-25 \%$ of whom use it on a daily basis. Voice word accuracy is improving rapidly and has more than a $90 \%$ accuracy for major platforms such as Apple's Siri or Amazon's Alexa.

\section{Trending topics}

So what are the key considerations for publishing professionals in an age of information overload, and a customer who is becoming increasingly tech-savvy and demanding?

\subsection{Competition}

- In the digital age, the competition is new and the rules have changed. Now everyone can be a publisher, and not just from a predatory publishing perspective. The new power players don't play by the rules and are challenging the boundaries of 'norm' in any number of industries:

- Airbnb is one of the biggest 'hoteliers,' but doesn't own any hotels

- Uber is one of the biggest taxi firms, but doesn't own any cars

- Facebook is the biggest publisher/media organization, but doesn't own or produce any content

Companies are continually expanding beyond their traditional roles to become publishers, and publishers are becoming tech companies (e.g. Wiley's acquisition of Atypon) and educators (e.g. RCNi (see: https://rcni.com/, TES Institute (see: https://www.tes.com/institute/). The platform players - Uber, Twitter, Airbnb, Etsy, LinkedIn, Facebook, Netflix and others - have built technology platforms that attract producers and consumers who interact on the platform to create value. This is about access, and not ownership. Furthermore, these players are driving the UX (user experience) standards to which traditional players must conform; they are driving the features and experience that users now take at face value and expect as a given throughout their online journeys. If that experience is lacking, they are lost for good.

Additionally, the competition is no longer similar companies within the same jurisdiction. As proven above, our competition comes from a range of areas - particularly tech - and we're now competing on a global scale, thus increasing the fight for attention among potential users and customers. While there isn't always specific communication with the audience, this doesn't mean that they are passive. If brands/publishers/content producers want to be heard above the noise on the internet, then they need to provide value. This should be:

- Informative

- Educational, and/or

- Entertaining

The customer is increasingly in charge of their online experience and relationship to a brand, and opportunity awaits for businesses that innovate and increase efficiency [6]. Mobility and flexibility are crucial to remain relevant.

\subsection{Mobile first}

Mobile is now the primary way in which users go online to access content/information; $79 \%$ of people aged 18-44 years have their smartphones with them twenty-two hours per day, interacting with their 
phones thirteen times per hour; and $80 \%$ of this cohort check their phones as soon as they wake up in the morning [2] - they are checking their social networks, their emails, the news. Never have we been more connected and 'switched on.'

It is imperative, therefore, that websites are mobile friendly. Google's algorithms were updated in 2016 to give precedence to mobile-optimized sites in search results. In fact, $60 \%$ of Google searches now come via mobile [10], and up to 53\% of visitors will leave a site if it doesn't load after three seconds (instant gratification is increasingly becoming the norm). Google has thrown a great deal of effort into its accelerated mobile pages (AMP) project, with the aim to 'make the web better for all.' While it isn't a tipping point for search results as yet, it can have huge implications on the discoverability and accessibility of content on mobile [9]:

- Pages generally load 4-times faster with AMP

- 700,000 domains are publishing AMP pages, including The Guardian, The New York Times, CNN, and Buzzfeed

- The Washington Post has found a 23\% increase in mobile search users visiting its content

Seventy-nine percent of consumers now use their smartphones for research purposes [4,9], and a growing proportion of these actually go on to complete purchases and sign up to newsletters or website accounts on their smartphones rather than waiting until they are at a desktop computer or laptop. These usage times peak during the morning and evening commutes to and from work, during lunch breaks and in the evening down time at home, during which many users now interact and shop on their smartphones/tablets while watching television. The customer journey is now a multi-platform journey, with users moving back and forth between devices to get things done; something that brands and marketers increasingly need to take into account.

\subsection{The rise of video}

Visual displays of information and data - through video in particular - are becoming increasingly important, as reflected in applications such as Instagram stories, Periscope, and Facebook Live. It is predicted that $75 \%$ of mobile (smartphone) traffic will be to video content by 2020 [1].

For those in the scientific and medical fields, video is gaining traction as it is 'easier to watch a video in the lab than to read a full journal article' [quote from an interviewee of the author's]. In fact, video is the key to user engagement, and the video we produce doesn't even need to be lengthy or particularly polished - Facebook Live, often recorded on mobile devices, has been found to gain ten times more engagement than standard video outputs.

Video has evolved significantly over the last century from linear formats (tune in or miss out) to ondemand (e.g. Netflix), semi-live (e.g. Snapchat stories) and real live (e.g. Facebook Live), and its use, sophistication, and relevance continues to grow rapidly [7]. While longer videos do have a value, for the transient web user, shorter videos will generally reap better engagement.

\subsection{Content ownership and social sharing}

Producing content is now no longer the mainstay of publishers and journalists; anyone can create a blog, write a post for LinkedIn or curate a Medium account (see: https://medium.com/. When the author was training as a journalist little more than a decade ago, citizen journalism https://medium.com was becoming a key tool through which newspapers could create stories: members of the public would share a photo directly with a newspaper or post onto social media, which a journalist would follow-up if 
newsworthy. This has moved to the next level in which journalists are actually competing with sparetime bloggers and content producers for attention - just think of any video blogger on YouTube with a huge following.

Equal to this, user-generated content remains a holy grail for website owners as the impact on a site's SEO ranking is significant, as well as enriching published content. Customers are becoming increasingly in control of their digital footprint, particularly on social media platforms, and are reaching greater levels of magnitude. Facebook has allowed content to become a lot more accessible and indeed, authentic. Many publishers are taking advantage of this and creating 'partnerships' with their subscribers, allowing them to create their own content on a publisher's site (e.g. Buzzfeed, The Guardian).

In research into user behaviours into the Future Science Group (FSG) Connect site, Neuro Central (www.neuro-central.com), we found that our users are avid social media users, particularly favouring LinkedIn, Facebook, and Twitter. Social sharing is now the primary way in which many people consume news and information, as well as a key tool through which authors can amplify their content. The development of tools such as Altmetric can give almost immediate and tangible data on the impact that an article has had. Social sharing also provides the opportunity for immediate feedback on content - if it is shareable then it is valuable.

Among Millennials, Facebook remains the most-used platforms, but image-sharing platforms such as Instagram are slowly catching up [8]. According to a report by Deloitte [3], photo sharing via social media or instant message rose from 5\% in 2015 to $12 \%$ in 2016, and is expected to continue rising as weekly photo-takers become daily photo-takers.

\section{Conclusions}

The digital world offers a plethora of solutions for publishers and authors to share and produce content, but as it continually develops it can be tricky to keep up. While the author has offered some key considerations in this article, they are by no means exhaustive, and the challenge for publishers and marketers is to know what the user intent is. If we are able to accurately predict how an individual user consumes content and navigates the digital space, then we will be able to ease the burden in an era of information overload.

\section{About the author}

Rosalind Hill joined Future Science Group in 2014 to grow its digital business. She is responsible for streamlining content and communicating it through a variety of platforms. She manages a team of editors, marketing, and sales staff. Hill has a BA in English Literature, a PGDip in Journalism, and a Diploma in Digital Business Leadership.

\section{References}

[1] Cisco Visual Networking Index: Global Mobile Data Traffic Forecast Update, 2016-2021, Cisco White Paper [published 7 February 2017; updated 28 March 2017]. Available from: http://www.cisco.com/c/en/us/solutions/collateral/ service-provider/visual-networking-index-vni/mobile-white-paper-c11-520862.html.

[2] Deloitte, There's no place like phone. Consumer usage patterns in the era of peak smartphone. Available from: https:// www.deloitte.co.uk/mobileuk/assets/pdf/Deloitte-Mobile-Consumer-2016-There-is-no-place-like-phone.pdf. 
[3] Deloitte, There's no place like phone. Consumer usage patterns in the era of peak smartphone. Available from: https:// www.deloitte.co.uk/mobileuk/assets/pdf/Deloitte-Mobile-Consumer-2016-There-is-no-place-like-phone.pdf, p. 35.

[4] M. Lawson, Overcoming Mobile Measurement Challenges to Drive Brand Lift and Sales Growth [published January 2017]. Available from: https://www.thinkwithgoogle.com/marketing-resources/data-measurement/ mobile-measurment-challenges-drive-sales-growth.

[5] M. Meeker, Internet Trends 2016, Code Conference [published 1 June 2016]. Available from: https://www.recode.net/ 2016/6/1/11826256/mary-meeker-2016-internet-trends-report.

[6] M. Meeker, Internet Trends 2016, Code Conference [published 1 June 2016]. Available from: http://www.kpcb.com/blog/ 2016-internet-trends-report, slide 40.

[7] M. Meeker, Internet Trends 2016, Code Conference [published 1 June 2016]. Available from: http://www.kpcb.com/blog/ 2016-internet-trends-report, slides 76-77.

[8] M. Meeker, Internet Trends 2016, Code Conference [published 1 June 2016]. Available from: http://www.kpcb.com/blog/ 2016-internet-trends-report, slide 93.

[9] C. Ratcliff, Accelerated Mobile Pages (AMP): one year on - stats and infographic [published 13 October 2016]. Available from: https://searchenginewatch.com/2016/10/13/accelerated-mobile-pages-amp-one-year-on-stats-and-infographic/.

[10] G. Sterling, Report: Nearly 60 percent of searches now from mobile devices [published 3 August 2016]. Available from: http://searchengineland.com/report-nearly-60-percent-searches-now-mobile-devices-255025. 